$\beta B$
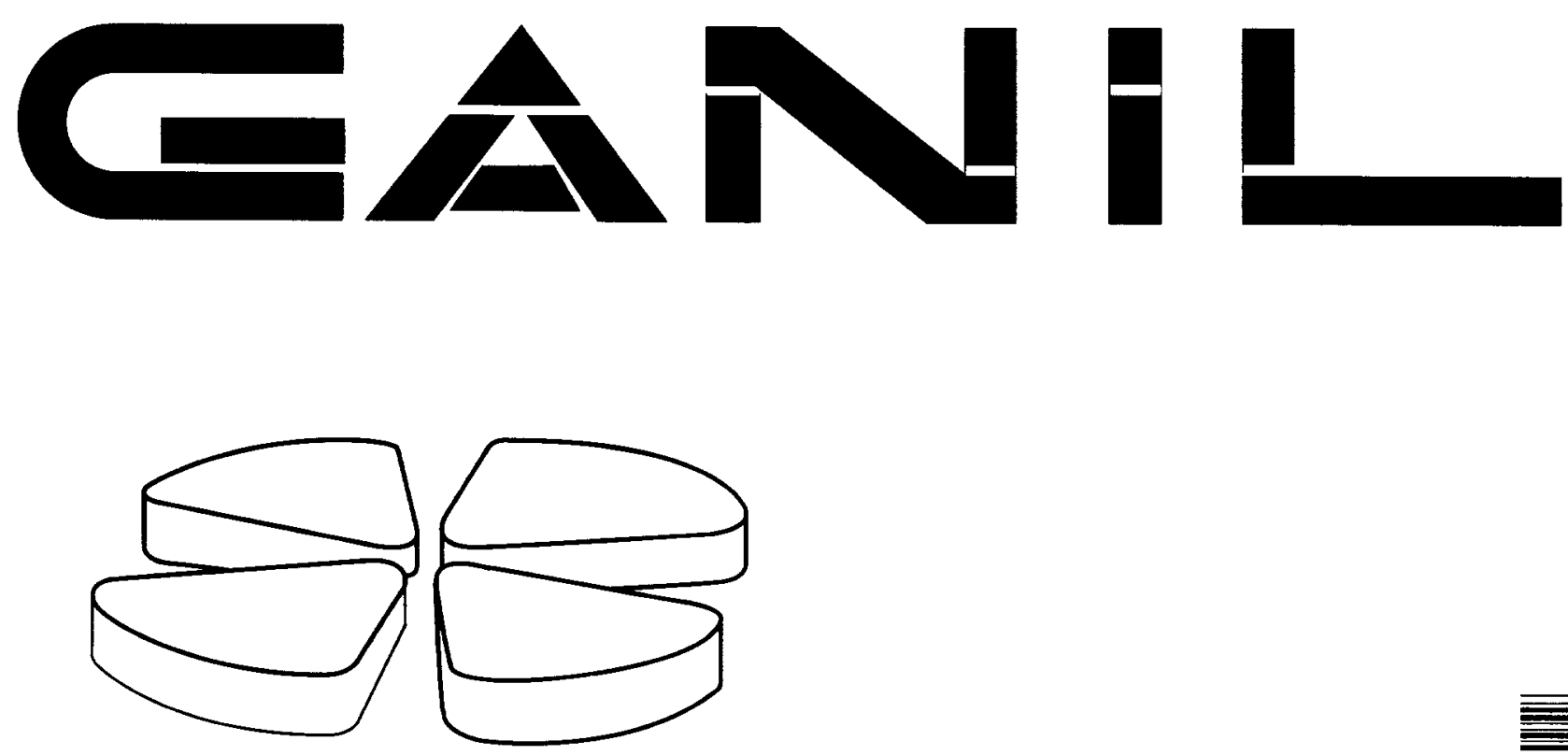

\title{
Anomalous Diffusion in Chaotic Scattering
}

T. Sroliowski ${ }^{\dagger}$ and M. Ploszajczak

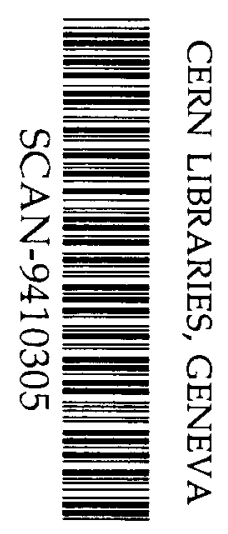

Grand Accélérateur National d'Ions Lourds (GANIL), BP 5027,

F-14021 Caen Cedex, France 



\title{
Anomalous Diffusion in Chaotic Scattering
}

\author{
T. Srokowski ${ }^{\dagger}$ and M. Ploszajczak \\ Grand Accélérateur National d'Ions Lourds (GANIL), BP 5027, \\ F-14021 Caen Cedex, France
}

\begin{abstract}
The anomalous diffusion is found for peripheral collision of atomic nuclei described in the framework of the molecular dynamics. Similarly as for chaotic billiards, the long free paths are the source of the long-time correlations and the anomalous diffusion. Consequences of this finding for the energy dissipation in deep-inelastic collisions and the dynamics of fission in hot nuclei are discussed.
\end{abstract}

PACS numbers: 24.60.Lz, 05.4.5.+b, 05.40.+j, 25.70.-z

t) On leave from Institute of Nuclear Physics, ul. Radzikowskiego 152, PL-31-342 Kraków, Poland 
Chaotic properties of atomic nucleus, well known from studies of compound nucleus resonances [1], have been recently discussed in connection with the heavy-ion fusion in the framework of molecular dynamics (MD) [2]. Regarded as a dynamical system, two colliding nuclei form a chaotic transient, i.e. they abide close together with the survival probability decaying exponentially in time. The system formed in this way can be interpreted as a compound nucieus. In particular, it has an exponential tail of the energy spectrum and the angular distribution of emitted particles is symmetric with respect to $\pi / 2[3]$. Those results come out of the MD model provided the collision is sufficiently violent to get both the fast memory loss and the equilibration, corresponding to the excitation of many overlapping resonances (Ericson fluctuations). as well as the Lorentzian shape of the energy autocorrelation function of an S-matrix element $C_{i j}(\epsilon)=\left\langle S_{i j}^{*}(E) S_{i j}(E+\epsilon)\right\rangle_{E}[4]$.

A different picture is expected if decay proceeds through a small number of isolated resonanses [5]. In this case, the scattering is nonhyperbolic and the survival probability is algebraic. Such a behaviour results from the stictiness of KAM tori existing in this case $[6]$. The decay is slow because trajectory must cross a hierarchy of cantori inhibiting the transport [7]. Both semi-classical and quantum calculations [4] show that the energy autocorrelation function $C(\epsilon)$ has the cusp at $\epsilon=0$, i.e. $d C(\epsilon) / d \epsilon \longrightarrow \infty$ as $\epsilon \longrightarrow 0$. It means that the fine scale fluctuations of the $S$ matrix are greatly enhanced. The presence of cantori affects also the velocity autocorrelation function $C(t)$ which falls slowlier than in purely chaotic case [8]. Transport through cantori can be described in the language of the diffusion process. Such a diffusion is anomalously enhanced, as has been found in modelling the two- 
dimensional solids [9]. In this case the variance of position $\sigma^{2}(t)$ rises with the time faster than linearly and the diffusion coefficient $D=\lim _{t \rightarrow \infty} \sigma^{2}(t) /(2 t)$ is infinite. One obtains also ' $1 / f$ ' power spectrum of velocity fluctuations. The anomalous diffusive behaviour relates to many physical problems of general application [10], also in the context of nuclear physics[11]. In MD approach, the anomalous diffusion could appear in peripheral collisions, because in this case the projectile and target preserve their identity for a long time, i.e. the correlations fall off slowly. This expectation is supported by the observation of the powerlaw decay $p(t) \sim t^{-z}$ with $z=4.56$ for the quasi-elastic reaction ${ }^{12} C+{ }^{12} C$ at angular momentum $l=16 \hbar$ and energy $E_{c m}=20 \mathrm{MeV}$ ) [3]. However, this value of the exponent $z$ is much larger than the typical value $1<z<2$ implied by the mechanism of transport through cantori [12]. It is also doubtful that such a chaotic and unstable system has any regular orbit to which trajectories could stick leading to the algebraic decay. The question therefore remains. What is the origin of this powerlaw survival probability?

In the following, we address this problem studying the diffusive features in the MD model of nuclear reactions for peripheral collisions. We will show that the motion in the phase space has indeed a diffusive component for large angular momenta and that, similarly as for chaotic billiards, there exist long-time correlations which are a source of both the algebraic decay and the anomalous diffusion. Hence, the transport features of $\mathrm{MD}$ for peripheral collisions do not require an existence of the KAM tori to explain the olsserved algebraic decay. We investigate also the consequences of the anomalous diffusion behaviour on the energy spectrum of reaction products and discuss the relation of this behaviour to deep-inelastic processes. 
Let us consider the collision of atomic nuclei consisting of some elementary constituents interacting via the wo-body Van-der-Waals - type potential with the attracting component, the coulomb tail and the strongly repulsive core at small distances. In the following. we regard $\alpha$-particles as such elementary constituents and take the potential from the adiabatic time-dependent Hartree - Fock calculations [13]. The ground state of both projectile and target is defined as a statistical ensemble, i.e. the phase space coordinates of all particles are sampled uniformly, taking into account the energy. linear momentum and angular momentum conservation [14]. Within this framework, various properties of $\alpha$-cluster nuclei (density distributions, binding energies. separation energies of $\alpha$-particles) are correctly reproduced [14]. The collision is represented by an ensemble of events with the same collective quantities, such as the relative energy and the impact parameter, but with the different internal coordinates.

For large impact parameter's we expect that the system will rotate like a molecule, slowly transferring the energy and particles (mass). A decisive factor in that process are violent collisions of particles at the border between the projectile and target nuclei, due to the repulsive core in the $\alpha-\alpha$ potential. The rest of the system continues its quasi-free, rotational motion. We also expect that the memory of initial conditions is kept for a long time. To check this assertion we calculate the velocity autocorrelation function $C^{\times}(t)=\langle\mathbf{v}(0) \cdot \mathbf{v}(t)\rangle$ for the reaction ${ }^{12} \mathrm{C}+{ }^{12} \mathrm{C}$ at $l=16 \hbar$ and $E_{c m}=20 \mathrm{MeV}$, where $\mathrm{v}(t)$ is the 18 -dimensional velocity vector and $<>$ means from now on the average over an ensemble of events. For such high angular momenta, one expects particularly small number of open decay channels[15] and, 
therefore, both the existence of algebraic survival probability[5] and the formation of long-lived orbiting complex! $10^{i} . C^{*}(t)$ oscillates with almost constant frequency but with diminishing amplitude (Fig. 1a). The oscillating pattern reflects the rotation in the space of collective variables. Since this rotation is somewhat trivial, we get rid of it redefining the autocorrelation function:

$$
\left.C_{i}\right)=C^{*}(t) \cos ^{-1}(\omega(t) t)
$$

The value of $\omega$, roughly equal 10 the collective angular velocity of the binary system, must be carefully chosen to avoid singularities. We have determined $\omega$ from extrema and zeros of $C^{*}(t)$, lineary 'xirapolating between them. Fig. 1b shows that $C(t) \sim$ $t^{-\gamma}$ with $\gamma=1$. The mean square displacement is given by the Green-Kubo formula $[17]:$

$$
\sigma^{2}(t) \equiv\left\langle(r(t)-r \cdot(0))^{2}\right\rangle=2 \int_{t_{0}}^{t}(t-\tau) C(\tau) d \tau \propto t \ln \frac{t}{t_{0}}-t+t_{0}
$$

Hence, the diffusion is anomalous and $D$ diverges logarithmically. The same dependence holds for mean-square displacement in the velocity space. The Fourier transform of $C(t)$ gives the power spectrum $S(\omega)$ which helps to decide about the nature of the transport. In on! case. $S(\omega) \sim|\ln \omega|$ and $S(\omega=0) \equiv 2 D=\infty$. In contrast to the $1 / f^{2}$ spectrum of a random walk, this spectrum is convergent when integrated to zero frequency and is divergent when integrated to infinite frequency. There is only a finite amount of power at low frequencies, but when we look at shorter time-scales the noise has no well-defined short-term mean. This resembles the features of the $1 / f$ spertrum of the 'flicker noise' which is also divergent when integrated to infinite frequency. though this divergence is weaker than in the case of 
the logarithmic spectrum. The same logarithmic power spectrum has been found for the Lorentz gas of hard dis - the extended Sinai billiard) [18]. The long-time correlations in that system originate from the long free paths of particles and not from the presence of regular struciures [19]. The same mechanism operates for peripheral collisions in MD. The system considered in this paper has the entire spectrum of positive Lyapunov exponents. Violent hard - core collisions between $\alpha$ - particles belonging to different nuclei. are necessary to transfer the energy from one nucleus to the other one. For large impact parameters such collisions are rare and most of the $\alpha$-particles are affected unly by weak interactions from the side of their neighbours in the same nucleus. In the sense of memory loss, this behaviour corresponds to the free motion in the Sinai billiard. During a long period of time, the collision of two nuclei resembles an intugrable, two-body system. In this case the hyperbolic instabilities, associated with heth homoclinic and heteroclinic connections [20], are not responsible for the dylinilical features of the system. Similarly, the Lyapunov exponents and the fractal dimensions do not determine the escape process and the survival probability is no longer exponential. The decay rate is affected by the long free paths. Thus, in spite of full chaoticity of the system, the peripheral collision mimics the nonhyperbolic saitering.

In the momentum space. diffusion causes the kinetic energy transfer from collective to internal degrees of freerlom. The internal kinetic energy $E_{k}^{(\text {int) }}$ can be defined as the mean-square displacement of the velocity vector with respect to its average $\left.\langle\mathbf{v}\rangle: E_{k}^{(i n t)}=\frac{m}{2}\langle(\mathbf{v}(t)-(\mathbf{v} t)\rangle)^{2}\right\rangle$ where $m$ is the mass of the $\alpha$-particle. The Green-Kubo formula (2) inplies that the energy dissipation rate is closely related 
to the autocorrelation function $C(t)$ :

$$
-d E / d t \propto \int_{t_{0}}^{t} C(\tau) d \tau
$$

The result of the evaluation of $E_{k}^{(i n t)}$ as the mean squared displacement of the velocity vector is presented in Fig. le. Most of the energy is rapidly dissipated in the entrance channel (the distance of the closest approach is reached at about $10^{-21} \mathrm{~s}$ ). For later times, according to (2), $E_{k}^{(i n t)}$ increases as:

$$
L_{k}^{(i n t)}=\alpha\left(t \ln \frac{t}{t_{0}}-t+t_{0}\right)
$$

where $\alpha$ is a constant. For the range of times considered in Fig. 1c, the deviation of calculated $E_{k}^{(i n t)}$ from the linear dependence on time is hardly visible. The finite and small available plase space cuts short the diffusion. For later times, (4) is no longer valid and $E_{k}^{(i n t)}$ saturates. Knowing the function $E_{k}^{(i n t)}(t)$ we are able to calculate the energy spectrum. Experimentally, the collective kinetic energy $E_{k}(t)=E_{k}\left(t_{0}\right)-E_{k}^{(i n t)}(t)$ can be measured when the system decays. For a given decay probability $p(t)$, combining $p(t)$ with $E_{k}(t)$, we get the energy spectrum :

$$
p(E(t))=\frac{p(t)}{(d E / d t)}=\frac{p(t)}{\alpha \ln \left(t / t_{0}\right)} .
$$

It is easy to see that $d p /\left.d E\right|_{E=E_{0}}=\infty$ and, therefore, the energy spectrum has the cusp at $E=E_{0}$. In fact. the formula (2) implies that the cusp behaviour is a generic property of diffusive systems, no matter what form of $p(t)$ is actually taken and how fast the correlations fall off.

For a fixed angular momentum, the predicted energy spectrum would appear as a peak positioned at rolatively low energy (a big energy loss) but with mass 
and atomic numbers of ejertiles similar to those of the projectile and target. An integration over angular monentum has a smoothing effect and broadens the peak. Such a picture is typical for drep-inelastic heavy-ion collisions. The detailed analysis of the shape of experimemial spectra and the comparison with (5) would in principle allow to determine the exponent $z$, giving informations about the lifetime of rotating systems. On the other hand. the dependence of the internal energy on the average lifetime can be measured utilizing experimental techniques of atomic and nuclear physics[21]. For near grazing collisions in light and medium heavy-ion nuclei, one has an evidence for an orhiting di-nuclear system which is formed after damping of initial energy and angular momentum and evolves through the exchange of nucleons $(\alpha$ - particles) into different configurations of a di-nuclear system[16] . The deepinelastic character of this processes is manifested also by an anomalous back-angle elastic scattering $[16,22]$.

The long-time rotation w weakly interacting reaction constituents is the necessary condition for the allomalous diffusion to take place. This condition is not fulfilled for central collisions. The correlation function in this case drops rapidly to zero (Fig. 2a) and the entire energy is dissipated at the very beginning of the reaction (Fig. 2b). The equilibration time is short, allowing the statistical properties of the compound nucleus to show up at the early stage of the reaction. Therefore, we predict two different nechanisms: the fusion dominated process at small angular momenta[2] with large number of open channels and the diffusive process associated with orbiting with small number of decay channels. The prediction of coexistence of these two mechanisms for light heavy-ion collisions is in agreement 
with the coupled-channel inal $\mid$ is $[23]$ and supported by experiments[24].

Fig. 1c indicates the presence of various mechanisms of excitation. When the diffusive process becomes vivible. most of the relative energy has been already dissipated in the vicinity of the point of the closest approach and could be described by a velocity-dependent frictinn force. However, the dissipative process at this stage is partially reversible, especially for the central collisions (see Fig. 2b). Such mixed elastoplastic behaviour of muclear matter, linking properties of elastic solids and viscous fluids, used to be attributed to the KAM tori in the phase space [25]. Since in the MD there are no regular structures, the present model shows that the elastoplastic behaviour can be obserred also in the fully chaotic systems. The dissipation due to the diffusion process, enurging at later times for peripheral collisions, is different. According to (3), its speed grows monotonically with time: the dissipation rate does not stabilize, as it would be the case for the normal diffusion, but instead it diverges logarithmically. The apparont irreversibility of the energy transfer can be related to the existence of the $1 / t^{\prime}-1$ iil of the correlation function. In this case the adiabatic approximation, corresponding 10 fully reversible processes, works worse than for a typical chaotic system with correlations falling off exponentially[26].

The divergence of the dissipation rate may have important consequences for the semi-phenomenological description of heavy-ion collisions and, in particular, hot fission process[21] . Going from central to peripheral collisions or from saddle to scission in fission process. one usually employs the same model of diffusion changing only the geometrical constraints such as the size of the 'window' or the deformation of the system. In view of the above results, this alone may not be sufficient as the 
change of the time scales incolred modifies the nature of the dissipation process. Consequently, for peripheral collisions or for strongly elongated shapes from saddle to scission, it is more appropriate to use the time- or coordinate- dependent diffusion coefficient. Fröbrich et al. $[2 i]$ have noticed that to describe the neutron multiplicities using the overdamperl Langevin equation, one has to increase the dissipation coefficient by about one orler of magnitude while going from high fissility systems (short fission-path length) to low fissility systems (long fission-path length). This observation finds a natural explanation in the anomalous diffusion mechanism for these strongly deformed shapes.

The similarity of the liffusive behaviour for systems as different as the MD and the Lorentz gas of hard liscs. follows from the fact that the powerlaw tail of the correlation function is determined only by the existence of long free paths and is totally insensitive 10 the details of the potential. Therefore, one expects that the above results will hold in a broad class of systems, including those of the Antisymmetrized Molecular Dynamics[28, 29] or Quantum Molecular Dynamics[30] . Investigations in this direction are in progress.

We are grateful to Y. Abe. G. Do Dang, A. Lépine-Szily and J. Okolowicz for useful discussions and suggestions. This work was partly supported by KBN Grant No. 2 P302 15704. 


\section{References}

[1] T. Brody, J. Flores. I. B. French. P. A. Mello, A. Panday and S. S. M Wong, Rev. Mod. Phys. 53. 325(1981).

[2] T. Srokowski, J. Okolowicz. S. Drożdż and A. Budzanowski, Phys. Rev. Lett. 71, 2867(1993).

[3] S. Drożdż, J. Okolowicz. T. Srokowski and A. Budzanowski, to be published.

[4] Y.-C. Lai, R. Blümel. E. Ott and C. Grebogi, Phys.Rev.Lett. 68, 3491 (1992).

[5] H. L. Harney, F.-M. littes and A. Müller, Ann. Phys. 220, 159(1992).

[6] S. Drożdz, J. Okolowicz and T. Srokowski, Phys. Rev. E 48, 4851(1993).

[7] J. D. Meiss and E. O11. Phrss. Rev. Lett. 55, 2741(1985).

[8] C. F. F. Karney. Physica D8, 360 (1983).

[9] T. Geisel, A. Zacherl and (. Radons, Phys. Rev. Lett. 59, 2503(1987);

T. Geisel, A. Zacherl and G. Radons, Z. Phys. B - Condensed Matter 71, $117(1988)$.

[10] J.-P. Bouchaud and A. Gieorges, Phys. Rep. 195, 127 (1990).

[11] D. Kusnezov, Phys. Rev. Lett. 72, 1990(1994).

[12] J. D. Meiss, Rev.Mod.Plyss. 64, 795 (1992).

[13] D. Provoost, F. Grünnı'r. K. Goeke and P.G. Reinhardt, Nucl. Phys. A431 , $139(1984)$ 
[14] K. Möhring. T. Srokowkik and D. H. E. Gross, Nucl. Phys. A533, 333(1991).

[15] R.M. Anjos, N. Added. N. Carlin, L. Fante, Jr., M.C.S. Figueira, R. Matheus, E.M. Szanto, C. Tereiru and Szanto de Toledo, Phys. Rev. C49, 2018 (1994).

[16] D. Shapira, R. Norotmy. I.D. Chan, K.A. Erb, J.L.C. Ford Jr., J.C. Peng and J.D. Moses, Phys. Let1. B114, 111 (1982).

[17] D.A. Mc Quarrie. Statislicul Mechanics ( Harper \& Row, New York, 1976).

[18] A. Zacherl, T. Geisel. .I. Nierwetberg and G. Radons, Phys. Lett. 114A, $317(1986)$.

[19] In fact, the KAM theorem does not apply to billiards which are fully chaotic.

[20] C. Jung and H. J. Scholz. J. Phys. A: Math. Gen. 20, 3607 (1987).

[21] D. Hilscher and H. Roninet. Ann. Phys. Fr. 17471 (1992).

[22] A. Lepine-Szily, J.M. (Oliveira Jr., P. Fachini, R. Lichtenthaler Filho, M.M. Obuti, W. Sciani. M.K. Steinmayer and A.C.C. Villari, Nucl. Phys. A539 (1992) $48 \pi$.

[23] C. Beck, Y. Abe, N. Aissauui, B. Djerroud and F. Haas, Phys. Rev. C49, 2618 (1994).

[24] A. Ray, D. Shapira. J. Giomez del Campo, H.J. Kim, C. Beck, B. Djerroud, B. Heusch, D. Blumenthal and B. Shivakumar, Phys. Rev. C44, 514 (1991).

[25] W. J. Swiatecki, Nucl.Ply's. A488, 375c (1988) 
[26] R. Brown. E. Ott and ('. (irebogi, Phys. Rev. Lett. 59, 1173 (1987);

R. Brown, E. Ott and ('. (irebogi, J. Stat. Phys. 49, 511 (1987).

[27] P. Fröbrich, I. I. Gontchall and N. D. Mavlitov, Nucl. Phys. A556, 281 (1993).

[28] S. Drożdz, J. Okolowič and M. Płoszajczak, Phys. Lett. B109, 145 (1982);

E. Caurier, B. Grammaticos and T. Sami, Phys. Lett. B109, 150 (1982);

W. Bauhoff, E. Caurier. B. Grammaticos and M. Płoszajczak, Phys. Rev. C32, $1915(1985)$.

[29] A. Ono, H. Horiuchi. T. Maruyama and A. Ohnishi, Phys. Rev. Lett. 68, 2898 (1992);

A. Ono, H. Horiuchi. T. Maruyama and A. Ohnishi, Prog. Theor. Phys. 87, 1185 (1992).

[30] J. Aichelin and H. Stöclier. Phys. Lett. B176, 14 (1986);

G. Peilert, H. Stöcker. W. Greiner, A. Rosenhauer, A. Bohnet and J. Aichelin, Phys. Rev. C39, 1402 (1919). 


\section{Figure captions}

Fig. 1

Temporal characteristics for 1 he grazing collision ${ }^{12} \mathrm{C}+{ }^{12} \mathrm{C}$ at $E_{c m}=20 \mathrm{MeV}$ and $l=16 \hbar$.

(a) The velocity autocorrelatiun function $C^{*}(t)$.

(b) The renormalized autocorrelation function $C(t)$ (see eq.(1)), where the trivial rotational motion has been removed. The dashed straight line denotes the function $1 / t$

(c) The internal kinetic energy calculated from the velocity variance. The initial energy corresponds to the encrgy of an internal motion in the ground state. The dashed straight line is plotted to guide the eye.

\section{Fig.2}

Temporal characteristics for the central collision ${ }^{12} \mathrm{C}+{ }^{12} \mathrm{C}$ at $E_{c m}=20 \mathrm{MeV}$ and $l=0$.

(a) The velocity autocorrelation function.

(b) The internal kinetic energy as a function of time. 

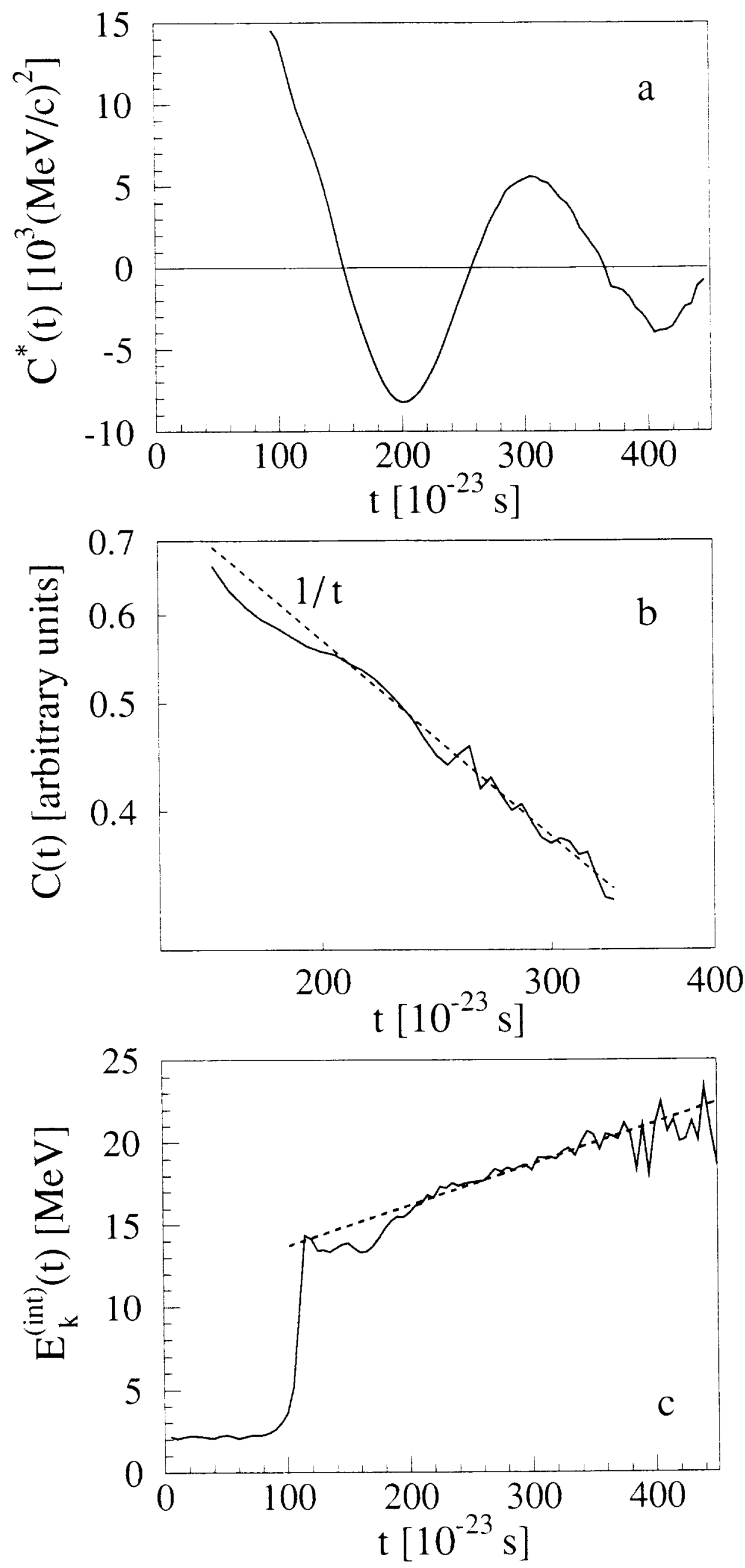

Fig. 1 

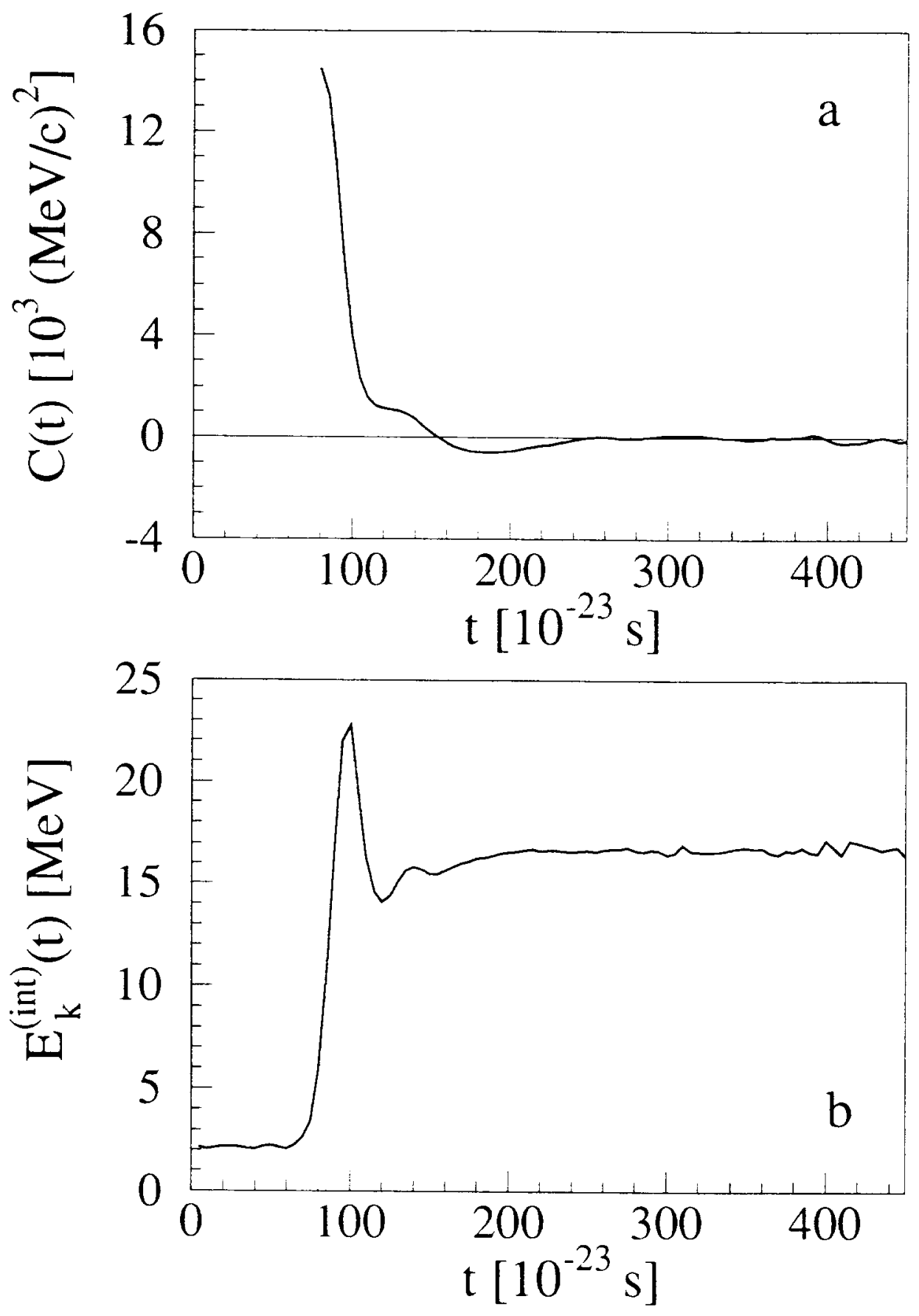

Fig. 2 